\title{
MODIFICATION OF FREEMAN-DURDEN DECOMPOSITION TO ELIMINATE THE NEGATIVE POWER PROBLEM
}

\author{
Akhmadiya Asset ${ }^{1,2}$, Nabiyev Nabi ${ }^{1}$, Moldamurat Khuralay ${ }^{2}$ \\ ${ }^{1}$ Kazakh Agro Technical University, Nur-Sultan, Kazakhstan \\ ${ }^{2}$ Eurasian National University, Nur-Sultan, Kazakhstan \\ (E-mail: ${ }^{1,2}$ aaset-04-77@mail.ru, ${ }^{1}$ n.nabiyev@kazatu.kz, ${ }^{2}$ moldamurat@yandex.kz)
}

\begin{abstract}
A new method in which completely eliminated the negative value of the scattering power is proposed in this paper. Primarily, here are presented the Freeman-Durden decomposition of the scattering powers, which are computed by using the coherency matrix elements with rotation and without rotation. Secondly, in this paper investigates the reasons of occurrence of negative values of the scattering powers. Then using the modification like in algorithm proposed by Yamaguchi for G4U, it was applied for the algorithm of Freeman-Durden decomposition to eliminate negative values. In this research, Radarsat-2 radar remote sensing data were used, which are acquired for study area Yushu County, Qinghai province, China. At the end, the comparison results of Yamaguchi G4U and a modified Freeman-Durden decompositions were presented.
\end{abstract}

Key words: Coherency matrix, Freeman-Durden decomposition, General fourcomponent decomposition with unitary transformation (G4U), scattering power, rotation.

\section{Introduction}

Many remarkable works were publicated on polarimetric decomposition such as decomposition of the target scattering matrix into three orthogonal components has been proposed by Cloude [1], Holm and Barnes [2], van Zyl [3], and Krogager. Cloude and Pottier have produced an excellent review of these decomposition theorems. Generally speaking, there are two types of decomposition, namely, coherent and incoherent decompositions [4]. Proposed by Freeman and Durden decomposition is model-based incoherent decomposition. The Freeman-Durden decomposition is a technique for fitting a physically based, three-component scattering mechanism model to the polarimetric SAR observations, without utilizing any ground truth measurements [4]. This decomposition based on the polarimetric coherency matrix as contribution of three scattering mechanisms: double-bounce, volume and surface scatterings. Later, it was introduced the fourth component of scattering mechanism proposed by Yamaguchi et al. [6], [7], [8]. From G4U (general four-component decomposition with unitary transformation) and subsequent modifications of the four-component decomposition is completely excluded negative values of the scattering powers [9]. However, there are problems of this kind in the original Freeman-Durden decomposition, mainly the 
appearance of a negative pixel values in double-bounce and surface scattering powers. It is sometimes called the negative power problem. The explanation of this problem is here in [10], [11]. This problem is solved partially, i.e., reduction the number or percentage of negative pixels by using a filtering or rotation in Freeman-Durden decomposition. Complete elimination of negative pixels is achieved through the replacement of negative values and the use of an algorithm of residual values derived from total power, as in the example G4U.

\section{Algorithm of the Freeman-Durden Decomposition}

The algorithm of Freeman-Durden decomposition based on model obtained from coherency matrix component as contribution three scattering mechanisms: surface, double-bounce and volume scattering [11]:

$$
[\boldsymbol{T}]=P_{s}[\boldsymbol{T}]_{\text {surface }}+P_{d}[\boldsymbol{T}]_{\text {double }}+P_{v}[\boldsymbol{T}]_{\mathrm{vol}}
$$

where $P_{s}, P_{d}$ and $P_{v}$ are power of each scattering components and their sum is equal to total power $(\boldsymbol{T P})$ :

$$
\boldsymbol{P}=P_{s}+P_{d}+P_{v}=T_{11}+T_{22}+T_{33}=T_{11}(\theta)+T_{22}(\theta)+T_{33}(\theta)
$$

where $T_{11}, T_{22}, T_{33}$ are diagonal elements of the coherency matrix, $T_{11}(\theta)$, $T_{22}(\theta), T_{33}(\theta)$ are diagonal elements of the rotated coherency matrix

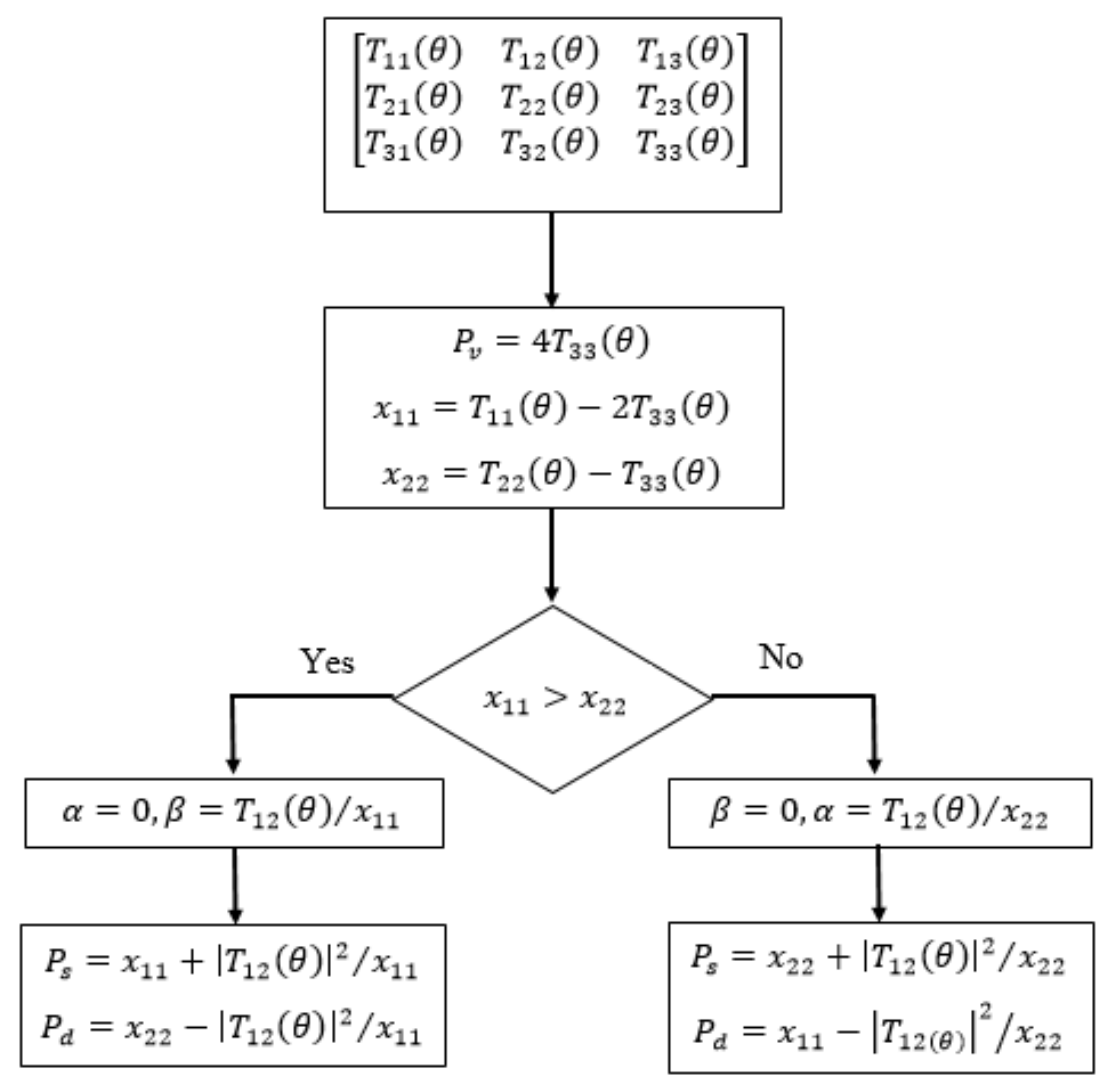

Figure 1: Algorithm of the Freeman-Durden Decomposition (FDD) with rotation of coherency matrix

After image processing according to this algorithm, there are obtained three components of the scattering powers $P_{s}, P_{d}$ and $P_{v}$. In this case, a first two 
components have negative pixel values, there is particularly prevalent in doublebounce scattering power, they are shown in Table 1.

Table 1. Percentage of pixels with negative $P_{S}$ and $P_{d}$

\begin{tabular}{|c|c|c|}
\hline & $P_{S}(\%)$ & $P_{d}(\%)$ \\
\hline Algorithm of FDD without rotation & 5.78 & 70.05 \\
\hline Algorithm of FDD with rotation & 6.88 & 50.93 \\
\hline Algorithm of proposed by Yang (2010) & 2.83 & 1.55 \\
\hline
\end{tabular}

The PolSAR data for this image processing acquired from Radarsat-2, the study area Yushu County, Qinghai province, China. The elements of the coherency matrix were generated in PolSARpro software. The remaining image processing was performed in ENVI software.

\section{Negative Power Problem}

From a mathematical point of view, the emergence of the negative value pixels in scattering powers is due to these three reasons:

$$
\left\{\begin{array}{l}
T_{11}<2 T_{33} \\
T_{22}<T_{33} \\
\left|T_{12}\right|^{2}>\left(T_{11}-2 T_{33}\right)\left(T_{22}-T_{33}\right)
\end{array}\right.
$$

The Freeman decomposition has been successfully applied to analyze the polarimetric data of the forest terrain. When applied to other types of terrain, however, $P_{S}$ or $P_{d}$ sometimes becomes negative. Since power is supposed to be nonnegative, the emergence of negative power values indicates that the model of the Freeman decomposition is not consistent with the actual scattering mechanism for these pixels. Note that in Table 1, the total number or percentage of negative pixels decreases after rotation of coherency matrix, but this is still to high to be ignored. A significant reduction has been achieved by using the algorithm of proposed Yang [11]. The reduction of percentage of negative pixels is possible mainly due to the decrease of $T_{33}$ and the increase of $T_{22}$ and $T_{11}$. However, a new modification taken from $\mathrm{G} 4 \mathrm{U}$ are proposed to perform complete elimination of negative pixels [9].

\section{Modification of Freeman-Durden Decomposition}

The total scattering power for three-component decomposition (2) are considered in this study. By using this equation, the modification and change of values scattering power and inequality situation are conducted according to Figure 2:

1) For volume scattering power $P_{v}$ : 
If $T P>P_{v}$ in this condition the volume scattering power is not changed $P_{v}^{m}=P_{v}$; another situation $T P<P_{v}$, volume scattering power is equal $P_{v}^{m}=T P$.

2) For surface and double-bounce scattering powers $P_{s}, P_{d}$ :

If TP $>P_{v}, P_{s} \geq 0$ and $P_{d} \geq 0$ in this condition the surface and double-bounce scattering powers are not changed $P_{s}^{m}=P_{s}, P_{d}^{m}=P_{d}$;

If $T P>P_{v}, P_{S} \geq 0$ and $P_{d} \leq 0$ in this case $P_{v}^{m}=P_{v}, P_{s}^{m}=T P-P_{v}^{m}, P_{d}^{m}=0$; If $T P>P_{v}, P_{s} \leq 0$ and $P_{d} \geq 0$ in this case $P_{v}^{m}=P_{v}, P_{s}^{m}=0, P_{d}^{m}=T P-P_{v}^{m}$; another situation $T P<P_{v}$ or $\left(P_{s} \leq 0\right.$ and $\left.P_{d} \leq 0\right)$ in this case $P_{v}^{m}=T P, P_{s}^{m}=$ $P_{d}^{m}=0$

Here, values before modification $\left(P_{s}, P_{d}\right.$ and $\left.P_{v}\right)$ after the algorithm of FreemanDurden decomposition and after modification $\left(P_{s}^{m}, P_{d}^{m}\right.$ and $\left.P_{v}^{m}\right)$ are existed. After such manipulations all values of the scattering powers become non-negative. Below, a comparison of the modified Freeman-Durden decomposition (MFDD) with G4U are presented in the form of RGB radar imageries (Figure 3).

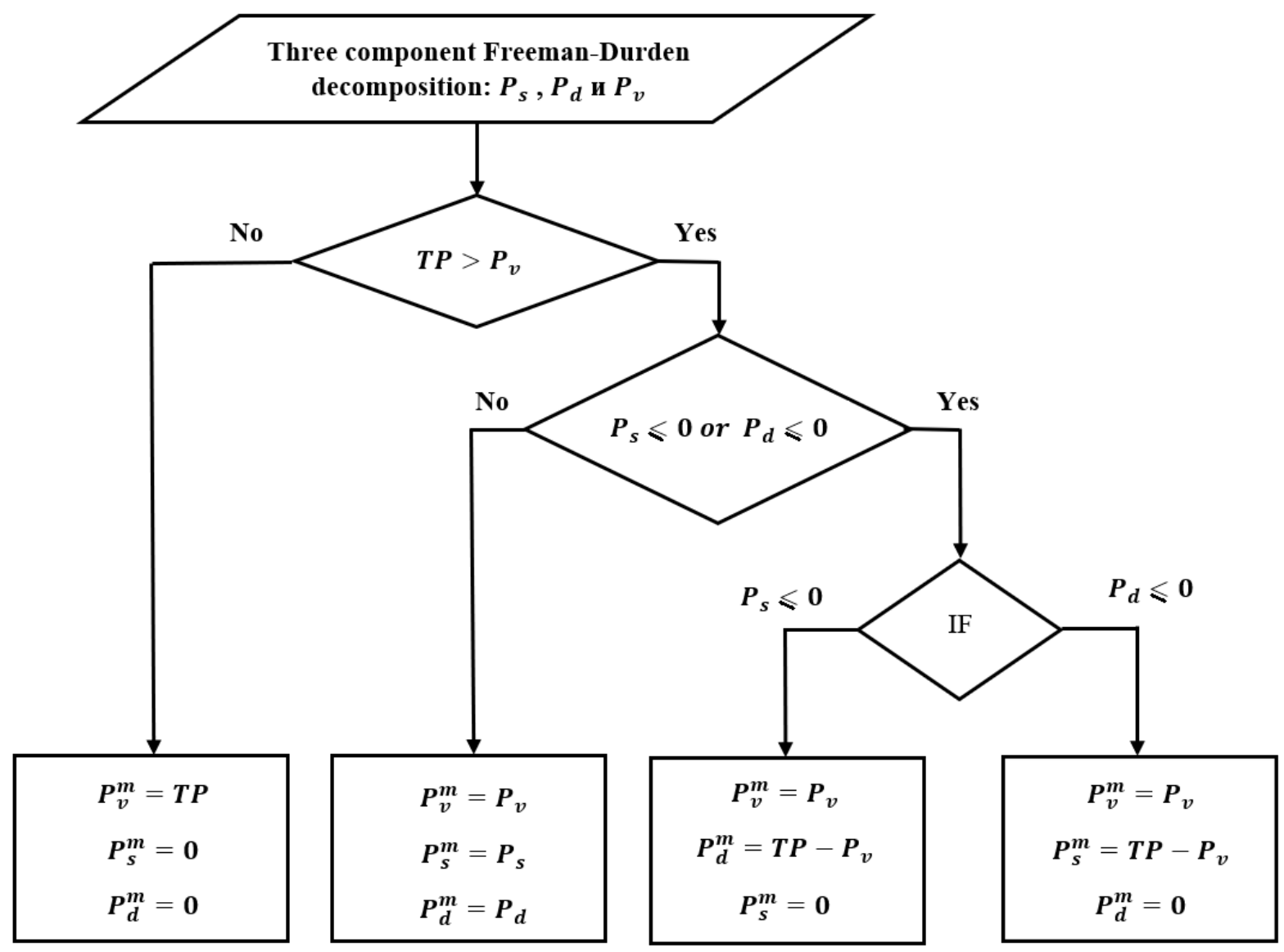

Figure 2: The algorithm for obtaining the modified Freeman-Durden decomposition 

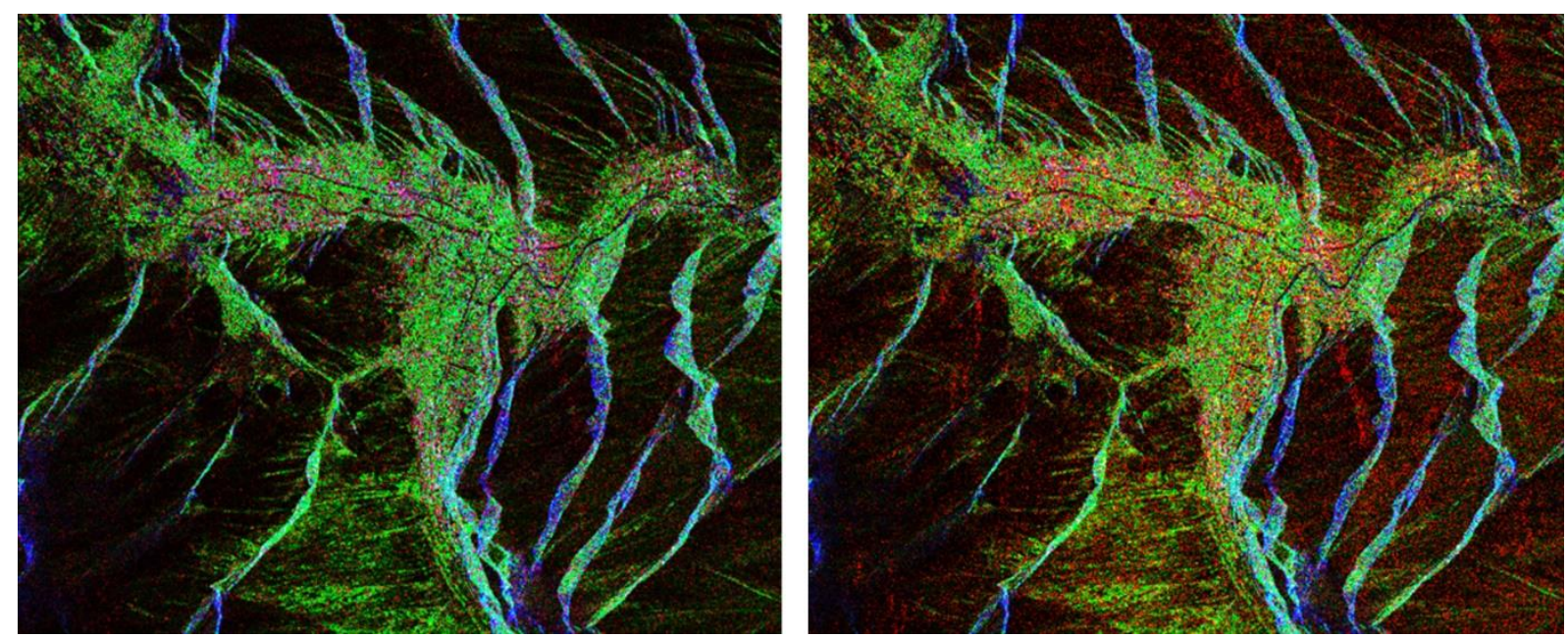

Figure 3: RGB Radar Imageries created from layers: Red - $P_{d}$, Green $-P_{v}$, Blue

- $P_{d}$. Left side: Modified Freeman-Durden decomposition (MFDD); Right side:

General four-component scattering power decomposition with unitary transformation $(\mathrm{G} 4 \mathrm{U})$ proposed by Yamaguchi.

\section{Conclusion}

This article, simply put, it is presented the method of exclusion of negative values of the scattering power, and recalculation of balances total power, as seen in the calculations we have three cases: single-component, two-component and threecomponent. Single-component appears under condition when the surface and double bounce scattering powers are zero, there is dominated volume scattering power. Two-component appears under condition when one of surface or double bounce scattering powers is zero, there are volume scattering power and one of surface or double bounce scattering powers. Three-component is ideal when all scattering powers are not equal to zero. Comparison of MFDD and G4U RGB images are characterized that in MFDD a little bit dominated by the color blue which means the surface scattering power, other differences are not visible. However, visual inspection shows a slight reduction in speckle-noise in the modified Freeman-Durden decomposition RGB image than in G4U RGB image (Figure 3). This alleged modification of Freeman-Durden decomposition will be checked by ground truth data, and also will be apply to other high-resolution PolSAR data (TerraSAR-X, Cosmo-SkyMed). The reasonableness of this approach still will be checked.

\section{References}

[1] Cloude, S. R. (1992) Uniqueness of target decomposition theorems in radar polarimetry. Direct and Inverse Methods in Radar Polarimetry. Proceedings of the NATO Advanced Research Workshop, 267-96 vol.1.

[2] Holm, W. A., R. M. Barnes (1988) On radar polarization mixed target state decomposition techniques., Proceedings of the 1988 IEEE National Radar Conference (Cat. No.88CH2572-6), 249-54. 
[3] van Zyl, J. J. (1993) Application of Cloude's target decomposition theorem to polarimetric imaging radar data., Proceedings of the SPIE - The International Society for Optical Engineering, 1748, 184-91.

[4] Cloude, S. R., E. Pottier (1996) A review of target decomposition theorems in radar polarimetry. Ieee Transactions on Geoscience and Remote Sensing, 34, 498-518.

[5] Freeman, A., S. Durden (1993) A three-component scattering model to describe polarimetric SAR data. Proceedings of the SPIE - The International Society for Optical Engineering, 1748, 213-24.

[6] Yamaguchi, Y., T. Moriyama, M. Ishido, H. Yamada (2005) Four-component scattering model for polarimetric SAR image decomposition. Ieee Transactions on Geoscience and Remote Sensing, 43, 1699-1706.

[7] Yamaguchi, Y., Y. Yajima, H. Yamada (2006) A four-component decomposition of POLSAR images based on the coherency matrix. Ieee Geoscience and Remote Sensing Letters, 3, 292-296.

[8] Yajima, Y., Y. Yamaguchi, R. Sato, H. Yamada, W.-M. Boerner (2008) POLSAR image analysis of wetlands using a modified four-component scattering power decomposition. Ieee Transactions on Geoscience and Remote Sensing, 46, 1667-1673.

[9] Singh, G., Y. Yamaguchi, S.-E. Park (2013) General Four-Component Scattering Power Decomposition With Unitary Transformation of Coherency Matrix. Ieee Transactions on Geoscience and Remote Sensing, 51, 3014-3022.

[10] Lee, J.-S., T. L. Ainsworth, Y. Wang (2014) Generalized Polarimetric Model-Based Decompositions Using Incoherent Scattering Models. Ieee Transactions on Geoscience and Remote Sensing, 52, 2474-2491.

[11] An, W., Y. Cui, J. Yang (2010) Three-Component Model-Based Decomposition for Polarimetric SAR Data. Ieee Transactions on Geoscience and Remote Sensing, 48, 2732-2739 